\title{
ARTICLE
}

Received 4 Jan 2013 | Accepted 21 May 2013 | Published 26 Jun $2013 \quad$ DOI: 10.1038/ncomms3040

\section{Coherent topological transport on the surface of $\mathrm{Bi}_{2} \mathrm{Se}_{3}$}

Dohun Kim¹, Paul Syers ${ }^{1}$, Nicholas P. Butch ${ }^{2}$, Johnpierre Paglione ${ }^{1} \&$ Michael S. Fuhrer ${ }^{1,3}$

The two-dimensional surface of the three-dimensional topological insulator is in the symplectic universality class and should exhibit perfect weak antilocalization reflected in positive weak-field magneto-resistance. Previous studies in topological insulator thin films suffer from high level of bulk $n$-type doping making quantitative analysis of weak antilocalization difficult. Here we measure the magneto-resistance of bulk-insulating $\mathrm{Bi}_{2} \mathrm{Se}_{3}$ thin films as a function of film thickness and gate-tuned carrier density. For thick samples, the magnitude of weak antilocalization indicates two decoupled (top and bottom) symplectic surfaces. On reducing thickness, we observe first a crossover to a single symplectic channel, indicating coherent coupling of top and bottom surfaces via interlayer tunnelling, and second, a complete suppression of weak antilocalization. The first crossover is governed by the ratio of phase coherence time to the inter-surface tunnelling time, and the second crossover occurs when the hybridization gap becomes comparable to the disorder strength.

\footnotetext{
${ }^{1}$ Department of Physics, Center for Nanophysics and Advanced Materials, University of Maryland, College Park, Maryland 20742-4111, USA. ${ }^{2}$ Condensed Matter and Materials Division, Lawrence Livermore National Laboratory, Livermore, California 94550, USA. ${ }^{3}$ School of Physics, Monash University, Victoria 3800, Australia. Correspondence and requests for materials should be addressed to M.S.F. (email: michael.fuhrer@monash.edu).
} 
W eak localization (WL) and weak antilocalization (WAL) describe corrections to the classical electrical conductivity of two-dimensional (2D) electron gases due to coherent interference of time-reversed paths. The 2D surface state of the three-dimensional strong topological insulator (TI) is fundamentally distinct from other $2 \mathrm{D}$ electron systems ${ }^{1}$ in that the Fermi arc encircles an odd number of Dirac points ${ }^{2-8}$. The TI surface is in the symplectic universality class and uniquely among 2D systems remains metallic and cannot be localized by (time-reversal symmetric) disorder ${ }^{9}$. In the absence of localization, the TI surface state is expected to exhibit perfect WAL described by, Hikami et al. ${ }^{10}$ :

$$
\Delta \sigma(H)=\alpha \frac{e^{2}}{\pi h}\left[\ln \frac{H_{0}}{H}-\psi\left(\frac{1}{2}+\frac{H_{0}}{H}\right)\right]
$$

where $H_{0}=\hbar / 4 D e \tau_{\varphi}$ is a characteristic field related to the phase coherence time $\tau_{\varphi}$ and diffusion constant $D, \psi$ is the digamma function, and $\alpha$ is the overall amplitude whose expected value for a single $2 \mathrm{D}$ channel is $1 / 2$. However, WAL is sensitive to the competition between the phase coherence time $\tau_{\varphi}$ and other time scales $^{11}$; in TI thin films, carriers may scatter into additional conducting channels (from top to bottom surface, or into the conducting bulk). Moreover, in finite-size samples inter-surface coupling can destroy the topological protection ${ }^{12-14}$, modifying the WAL behaviour. The question arises: at what size can a thin TI sample be treated as having decoupled topological surface states?

In contrast to previous studies of WAL in TI thin films ${ }^{15-22}$ where strong surface to bulk scattering dominates inter-channel coupling, here we report WAL measurements in gate-tuned, bulkinsulating $\mathrm{Bi}_{2} \mathrm{Se}_{3}$ thin films where we expect negligible surfacebulk scattering. We find that the WAL behaviour in TI regime is governed by the ratio of $\tau_{\varphi}$ to the inter-surface tunnelling time $\tau_{\mathrm{t}}=h / 2 \Delta$, where $h$ is Planck's constant and $\Delta$ is the hybridization gap induced by inter-surface tunnelling ${ }^{12,14}$. For thick films $(17 \mathrm{~nm}), \tau_{\varphi} / \tau_{\mathrm{t}}<1$ and we observe the signature of a true $2 \mathrm{D}$ topological metal: perfect WAL according to equation (1) with $\alpha=1 / 2+1 / 2=1$ corresponding to two decoupled TI surfaces each with $\alpha=1 / 2$. A thickness and doping-dependent crossover is
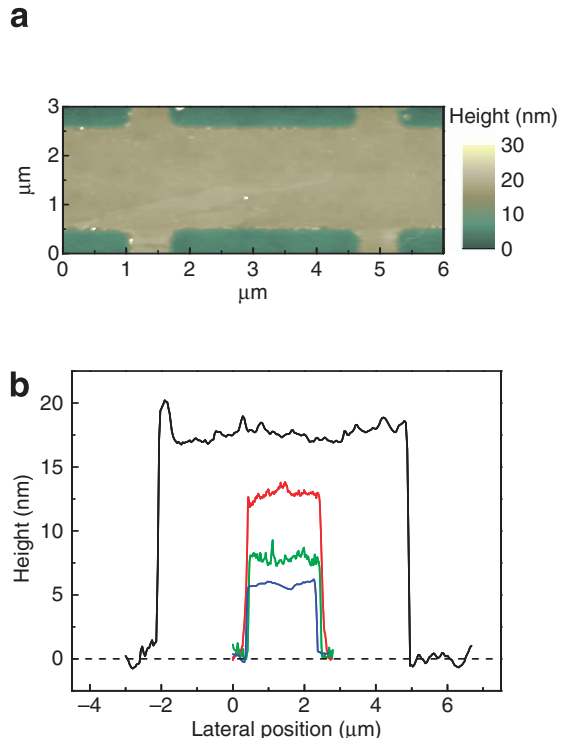

observed when $\tau_{\varphi} / \tau_{\mathrm{t}}>1$ to a regime where $\alpha=1 / 2$, indicating the coherent coupling of two TI surfaces. The result indicates that WAL is extraordinarily sensitive to sub-meV coupling between top and bottom topological surfaces, and the surfaces of a TI film may be coherently coupled even for thicknesses as large as $12 \mathrm{~nm}$. For even thinner samples, $\Delta$ becomes comparable to the disorder strength and the Berry's phase is completely randomized at low carrier density causing the suppression of WAL and WL, reflecting the loss of topological protection for strongly coupled surfaces.

\section{Results}

Characterization of $\mathbf{B i}_{2} \mathrm{Se}_{3}$ Hall bar devices. We study mechanically exfoliated $\mathrm{Bi}_{2} \mathrm{Se}_{3}$ single crystals ${ }^{23}$ ranging in thickness from 5 to 17 quintuple layers (QL). Figure 1a shows a representative device (thickness $12 \mathrm{QL}$ ), where height profiles across the width direction of the 17, 12, 7 and $5 \mathrm{QL}$ devices are shown in Fig. 1b. In order to achieve the topological transport regime, we employed molecular charge transfer $p$-type doping by thermal evaporation of 2,3,5,6-tetrafluoro-7,7,8,8-tetracyano quinodimethane, described previously ${ }^{24}$ (see also Methods, Supplementary Fig. S1, and Supplementary Note 1).

Figure $1 c, d$ show the longitudinal resistivity $\rho_{\mathrm{xx}}$ and Hall carrier density $n_{\mathrm{H}}=1 /\left(e R_{\mathrm{H}}\right)$ (where $R_{\mathrm{H}}$ is the Hall coefficient, and $e$ is the elementary charge) of the devices at a temperature of $2 \mathrm{~K}$ as a function of back gate voltage $V_{\mathrm{g}}$. We measure $n_{\mathrm{H}}$ in the range of $2-7 \times 10^{12} \mathrm{~cm}^{-2}$ at zero gate voltage. The carrier density in the topological surface state at the bulk conduction band edge is $\sim 0.8-1.0 \times 10^{13} \mathrm{~cm}^{-2}$, hence the devices are close to the TI regime before application of a back gate voltage. Ambipolar electric field effects are indicated by the sharp peak of $\rho_{\mathrm{xx}}\left(V_{\mathrm{g}}\right)$ and the sign change in $n_{\mathrm{H}}$ at the charge neutrality points $\left(V_{\mathrm{g}, 0}\right.$, Fig. $1 \mathrm{c}$ dashed lines). The dependence of $\rho_{\mathrm{xx}}\left(V_{\mathrm{g}, 0}\right)$ on temperature $T$ in all of our devices, including $5 \mathrm{QL}$, shows metallic behaviour (see Supplementary Fig. S2), which is likely due to conduction through inhomogeneity-driven electron-hole puddles ${ }^{24,25}$. However, the thinnest (5QL) device shows an anomalously large maximum $\rho_{\mathrm{xx}}\left(V_{\mathrm{g}, 0}\right)$ of about $23 \mathrm{k} \Omega$, which cannot be understood within the self-consistent theory for a Dirac band in the

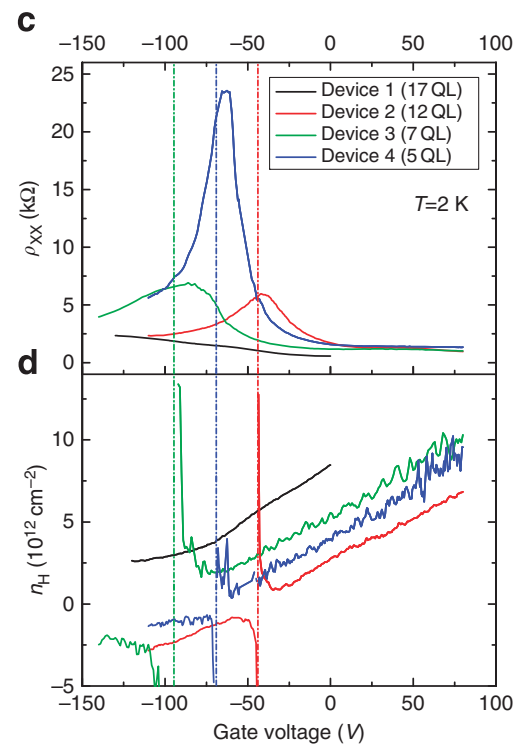

Figure 1 | Characterization of $\mathbf{B i}_{\mathbf{2}} \mathbf{S e}_{\mathbf{3}}$ Hall bar devices. (a) Atomic force microscopy image of $12 \mathrm{QL} \mathrm{Bi}_{2} \mathrm{Se}_{3}$ Hall bar device. (b) Height profiles along width directions of the devices used in this study. (c) Longitudinal resistivity $\rho_{\mathrm{xx}}$ and (d) sheet carrier density $n_{H}$ determined from Hall measurement as a function of back gate voltage at the temperature of $2 \mathrm{~K}$ for 17 (black), 12 (red), 7 (green) and 5 (blue) QL devices. The dashed lines show charge neutrality points. 
presence of charge disorder ${ }^{24,26,27}$, suggesting that the intersurface hybridization gap $\Delta$ becomes important in determining the resistivity in this regime ${ }^{12}$. We note that true insulating behaviour (divergent $\rho_{\mathrm{xx}}$ as $T \rightarrow 0$ ) is not observed for the $5 \mathrm{QL}$ sample, but was previously observed for $3 \mathrm{QL} \mathrm{Bi}_{2} \mathrm{Se}_{3}$ (ref. 13). More work is needed to understand the $\rho_{\mathrm{xx}}$ maximum in the $5 \mathrm{QL}$ sample (see Supplementary Note 2 for further discussion).

WAL in the topological transport regime. We now turn to discuss gate-tuned WAL behaviour in the TI regime. Figure 2 shows the magneto-conductivity $\Delta \sigma(H)$ for all four devices. Curves are taken at similar carrier density $n=C_{\mathrm{g}}\left(V_{\mathrm{g}}-V_{\mathrm{g}, 0}\right) / e$ (where gate capacitance $C_{\mathrm{g}} \approx 11 \mathrm{nFcm}^{-2}$ ) ranging from $\approx 7 \times 10^{12} \mathrm{~cm}^{-2}$ ( $n$-type) to $-2 \times 10^{12} \mathrm{~cm}^{-2}$ (p-type) except for the $17 \mathrm{QL}$ device where only $n$-type carrier density $(7$ and $2 \times 10^{12} \mathrm{~cm}^{-2}$ ) could be observed due to relatively high initial doping. The data for the entire range of $n$ and thickness can be fitted (dashed curves in Fig. 2) well with equation (1). The validity of equation (1) in the multichannel limit has been addressed in a number of previous studies ${ }^{16,19,20,28}$, finding that equation (1), in particular the logarithmic correction term, provides a robust physical description of WAL behaviour even in the multichannel limit irrespective of the Drude conductivity of each channel ${ }^{20,28}$. Therefore, $\alpha=m / 2$ probes the number of channels $m$. Moreover, although crossover to WL was predicted ${ }^{29}$ in a gapped TI thin films (considered single 2D channel), further study of WAL in multi-channels including coherent coupling between channels ${ }^{28}$, as well as experimental observation in molecular beam epitaxy grown $\mathrm{Bi}_{2} \mathrm{Se}_{3}$ (ref. 22) points out that WL can be neglected down to $\sim 5$ QL thickness. Our samples thus lie in the regime of dominant WAL.

Coupling of coherent transport in $\mathrm{Bi}_{2} \mathrm{Se}_{3}$ surface states. Figure 3 shows the variation of $\alpha$ obtained from the fit to equation (1) as a function of $n$ for 17 (black), 12 (red), 7 (green) and 5 (blue) QL devices measured at $2 \mathrm{~K}$. In all devices $\alpha$ is close to $1 / 2$ at high $n \approx 8 \times 10^{12} \mathrm{~cm}^{-2}$, which we interpret as WAL in a single strongly coupled coherent channel ${ }^{15-17,19,20}$ in the presence of surface to bulk scattering, as bulk states becomes energetically accessible (non-TI regime). Thus, we restrict the carrier density ranging $n<6 \times 10^{12} \mathrm{~cm}^{-2}$ for investigating WAL behaviours in TI regime. The dependence of $\alpha$ on gate tuning shows very different behaviours depending on the thickness of the devices. The thickest device (17 QL) shows WAL consistent with two decoupled top and bottom topological surfaces $(\alpha \approx 1)$ starting at moderate $n \approx 6 \times 10^{12} \mathrm{~cm}^{-2}$, maintained down to the minimum accessible $n$. Similar variation of $\alpha$ ranging from 0.7 to 1 was observed in Steinberg et al. ${ }^{20}$, with the application of top gate, and interpreted there as the decoupling of top surface and bulk (plus bottom) channels due to formation of bandbending-induced depletion layer. We do not rule out the possible contribution of bulk-surface scattering in $17 \mathrm{QL}$ at high $n$. However, we note that this effect alone cannot explain the general behaviour of $\alpha$ for low $n$ and thinner devices. Notably, we identify two crossovers in the $12 \mathrm{QL}$ device: we observe sharp transition of $\alpha$ from $\approx 1 / 2$ to $\approx 1$ near $n \approx 1 \times 10^{12} \mathrm{~cm}^{-2}(n$-type), and back to $1 / 2$ at $n \approx-1 \times 10^{12} \mathrm{~cm}^{-2}$ ( $p$-type). $\Delta \sigma(H)$ in our devices include a moderate contribution from (universal) conductance fluctuations (Fig. 2), as commonly observed in micro-fabricated $\mathrm{Bi}_{2} \mathrm{Se}_{3}$ devices ${ }^{16,21}$; the fluctuations are reduced by fitting to equation (1) over a large field range $(0 \mathrm{~T}, 0.8 \mathrm{~T})$. At the upper end of this field range, the magnetic length $l_{\mathrm{B}}=(\hbar / e H)^{1 / 2}$ becomes comparable to the electronic mean free path $l_{\mathrm{mfp}}$, approaching the regime where equation (1) may not be valid. However, we verified that the effect of increasing the field fitting

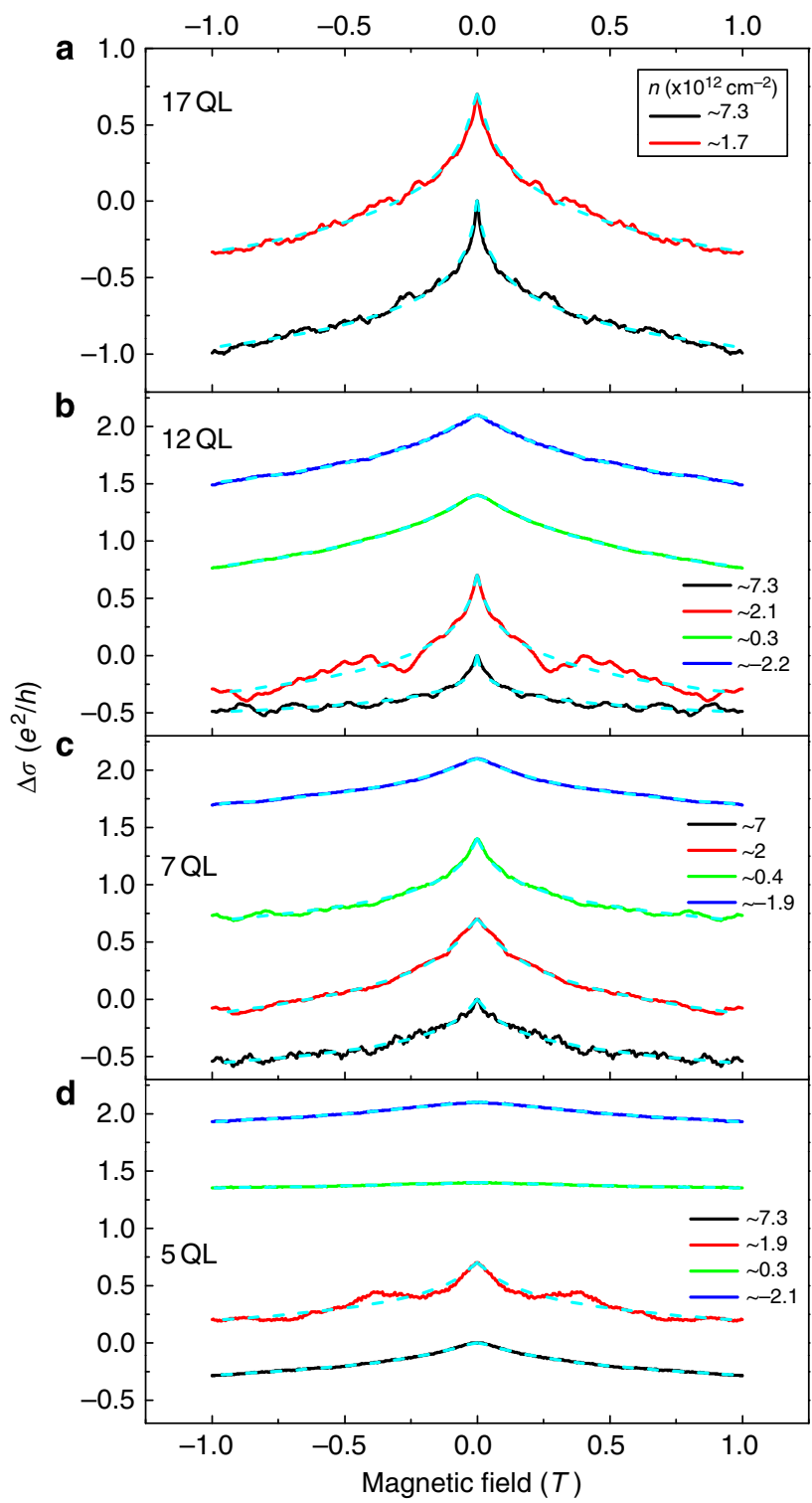

Figure 2 | WAL in the $\mathbf{T I}$ regime. Magneto-conductivity $\Delta \sigma$ as a function of perpendicular magnetic field $H$ in (a), 17, (b), 12, (c), 7 and (d), $5 \mathrm{QL}$ devices measured at $2 \mathrm{~K}$ at gate-induced carrier densities indicated in the legends. Dashed curves show least-square fits to equation (1). Zeros of all curves are offset by $0.7 \mathrm{e}^{2} / \mathrm{h}$ for clarity.

range from $(0 \mathrm{~T}, 0.02 \mathrm{~T})$, where $l_{\mathrm{B}}>l_{\mathrm{mfp}}$, to $(0 \mathrm{~T}, 1 \mathrm{~T})$, where $l_{\mathrm{B}} \gtrsim l_{\mathrm{mfp}}$, is only to reduce the fluctuations in $\alpha$, without changing the average value, and that conductance fluctuations result in variation in $\alpha<0.1$ in a given thermal run (see Supplementary Fig. S3c and Supplementary Note 3). Moreover, we repeated similar WAL measurements at $2 \mathrm{~K}$ for the $12 \mathrm{QL}$ device on five different thermal runs, where the conductance fluctuation contribution is randomized, and find that the crossovers are reproducible within the experimental uncertainty represented by the standard deviation determined from the repetitive measurements (Fig. 3, error bars). This transition is absent in the 7 QL device, where WAL in the entire range of $n$ indicates a strongly coupled single channel $(\alpha \approx 1 / 2)$. Finally, at an even smaller thickness $(5 \mathrm{QL})$, we observe strong suppression of WAL for $-1 \times 10^{12} \mathrm{~cm}^{-2}<n<1 \times 10^{12} \mathrm{~cm}^{-2}$, (inset of Fig. 3, see also Fig. 2d). 


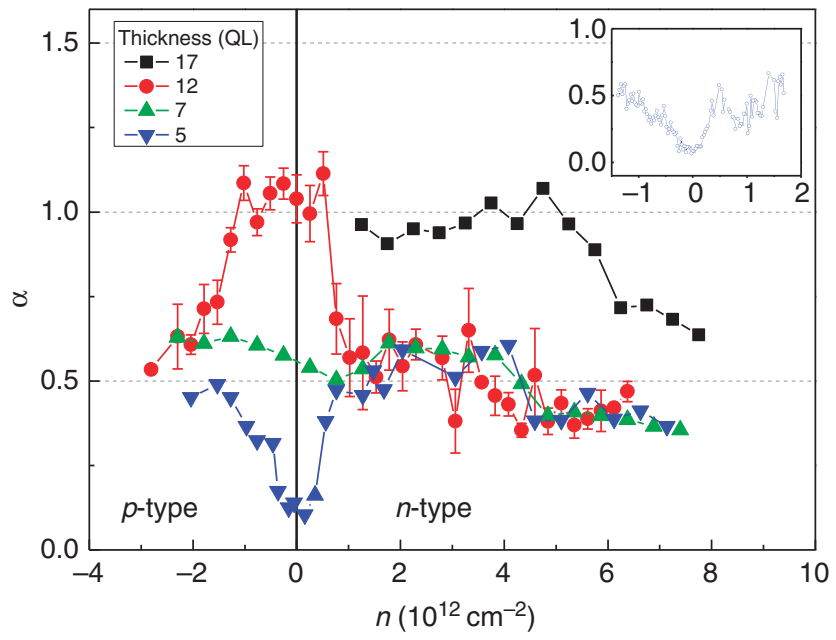

Figure 3 | Coupling of coherent transport in $\mathrm{Bi}_{2} \mathbf{S e}_{3}$ surface states. Variation of the amplitude of WAL $\alpha$ as a function of 2D carrier density $n$ for 17 (black square), 12 (red circle), 7 (green triangle) and $5 \mathrm{QL}$ (blue triangle) thick devices measured at $2 \mathrm{~K}$. For the $12 \mathrm{QL}$ device, the error bars show standard deviations determined from WAL measurements taken at five different thermal runs. The inset shows detailed behaviour of suppression of WAL for $5 \mathrm{QL}$ at small $n$.

Phase coherence time versus inter-surface tunnelling time. We now examine the coherence time $\tau_{\varphi}$ and compare with the estimated interlayer tunnel time $\tau_{\mathrm{t}}$. We estimate $\tau_{\varphi}=\hbar / 4 e D H_{0}$ from the fits to equation (1) and using $D=\tau v_{\mathrm{F}}^{2} / 2$ where Fermi velocity $v_{\mathrm{F}} \approx 3 \times 10^{7} \mathrm{~cm} \mathrm{~s}^{-1}$ for $\mathrm{Bi}_{2} \mathrm{Se}_{3}$ (ref. 30), and the momentum relaxation time $\tau$ is calculated from the measured $\sigma(n) . \tau_{\mathrm{t}}$ represents the characteristic time of transition (half the period Rabi oscillations) between localized states in two quantum wells with energy splitting $\Delta$, thus $\tau_{\mathrm{t}}=h / 2 \Delta$. We estimate $\Delta$ in $\mathrm{Bi}_{2} \mathrm{Se}_{3}$ by fitting the existing experimental data ${ }^{12}$ to an exponentially decaying function. We estimated the range of $\Delta(t)$ by including (1) sizable $\Delta$ in $2,3,4$ and $5 \mathrm{QL}$ (upper bound) and (2) all data from 2 to $8 \mathrm{QL}$ (lower bound) in the fit and obtained $\Delta(t)=[992 \mathrm{meV}] e^{-0.67[t(\mathrm{~nm})]}$ and $\Delta(t)=$ $[879 \mathrm{meV}] e^{-0.62[t(\mathrm{~nm})]}$. The resultant range of $\Delta$ is $34.8-39.5$, 9.1-11.44, $0.32-0.51$ and $0.01-0.02 \mathrm{meV}$ for $5,7,12$ and $17 \mathrm{QL}$, respectively. (Theoretical calculations of finite-size effects in $\mathrm{Bi}_{2} \mathrm{Se}_{3}$ predict systematically smaller $\Delta$ at a given thickness and also suggest that $\Delta$ shows oscillatory decaying behaviour ${ }^{14}$, which do not seem to be observed in the previous work ${ }^{12}$ or this work.)

Figure 4 shows the estimated $\tau_{\varphi}$ (symbols) and $\tau_{t}$ (hatched areas) as functions of $n$ for the different thickness samples. Upon gate tuning, $\tau_{\varphi}$ changes by an order of magnitude ranging from $\approx 4$ to $\approx 50 \mathrm{ps}$, and shows a sharp dip near $n=0$. For the $17 \mathrm{QL}$ device, $\tau_{\varphi} / \tau_{\mathrm{t}}<1$ in the entire range of $n$. In this limit, electrons on either surface lose coherence before scattering to the other, thus each surface acts as an independent coherent transport channel and $\alpha \approx 1$. In contrast, a crossover occurs from $\tau_{\varphi} / \tau_{\mathrm{t}}>>1$ at high unipolar $n$ - and $p$-type carrier densities to $\tau_{\varphi} / \tau_{\mathrm{t}}<1$ near $n=0$ in the $12 \mathrm{QL}$ device, which is consistent with the observed crossover from $\alpha=1 / 2$ to $\alpha=1$ (Fig. 3). In the thinner device (7 QL), the condition $\tau_{\varphi} / \tau_{\mathrm{t}} \gg 1$ is satisfied, and the two surfaces are strongly coupled $(\alpha \approx 1 / 2)$ for the entire range of $n$. The systematic tuning from coherent coupled to decoupled regimes upon variation of $\tau_{\varphi} / \tau_{\mathrm{t}}$ confirms that interlayer tunnelling is the primary coherent coupling mechanism in the TI regime. As $\tau_{t}$ is primarily determined from the thickness of $\mathrm{Bi}_{2} \mathrm{Se}_{3}$ thin films, whereas $\tau_{\varphi}$ can vary significantly depending on the sample quality, we expect

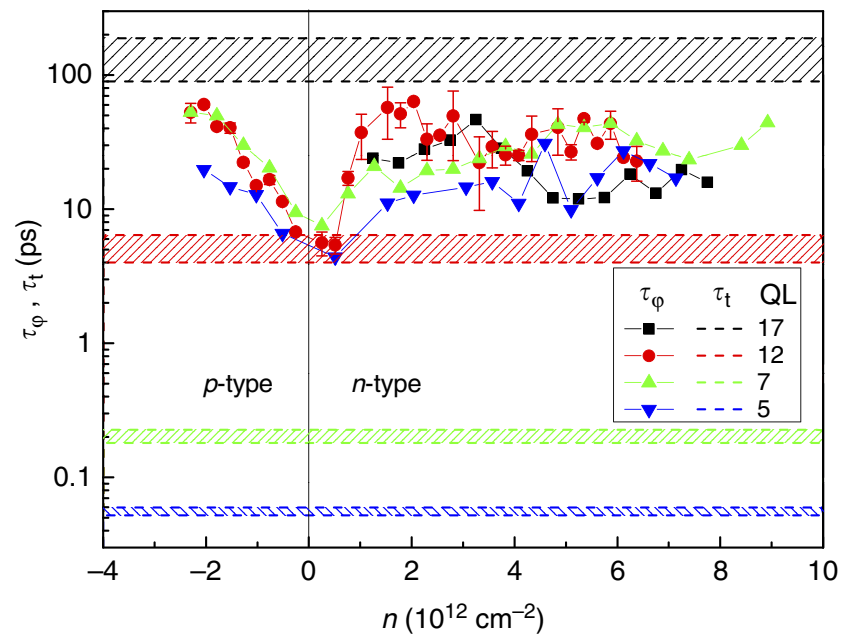

Figure 4 | Phase coherence time versus interlayer tunnelling time. Comparison of phase coherence time, $\tau_{\varphi,}$ determined from fit to equation (1) and transport scattering time to interlayer tunnelling time, $\tau_{\mathrm{t}}$ estimated from surface hybridization induced energy gap $\Delta$ as a function of $2 \mathrm{D}$ carrier density $n$. Symbols are experimentally measured $\tau_{\varphi}$ for 17 (black square), 12 (red circle), 7 (green triangle) and 5 QL (blue triangle) devices. The hatched areas with corresponding colours show estimated windows of inter-surface tunnelling time $\tau_{t}$.

that the decoupling crossover thickness is not universal but can occur in different thickness if the surface phase coherence can be modified. For instance, decreasing phase coherence of the surface electrons by addition of magnetic impurities ${ }^{31}$ should result in a reduced critical thickness above which decoupled coherent transport can be observed. In principle, temperature-dependent dephasing offers an additional opportunity to tune from coupled to decoupled surfaces. However, we observe weak dependence of the coherence time on temperature (see Supplementary Fig. S4 and Supplementary Note 4), which limits temperature as a parameter to control interlayer coherence. The weak temperature dependence of $\tau_{\varphi}$ is observed elsewhere ${ }^{21}$ but not understood, though it suggests an extrinsic mechanism. If the lowtemperature decoherence can be improved, it may be possible to explore temperature-driven coherent coupling/decoupling of the TI surfaces.

The behaviour described above is in general consistent in the 5 QL device except near the Dirac point, where WAL is strongly suppressed. The suppression of WAL can be understood in terms of the expected change of Berry phase $\theta_{\mathrm{B}}=\pi\left(1-\Delta / 2 E_{\mathrm{F}}\right)$ in TIs $s^{29,32}$ as a function of $\Delta$ and the Fermi energy $E_{\mathrm{F}} ; \theta_{\mathrm{B}}$ is reduced from $\pi$ when hybridization induced gap opens. Assuming a gapped Dirac dispersion $E_{\mathrm{F}}=\sqrt{\left(\hbar v_{F} k_{F}\right)^{2}+(\Delta / 2)^{2}}$, we estimate $E_{\mathrm{F}} \leq 52 \mathrm{meV}$ and $\theta_{\mathrm{B}} \leq 0.67 \pi$ at $|n| \leq 1 \times 10^{12} \mathrm{~cm}^{-2}$. This range of $n$ is comparable to the electron-hole puddle density $n^{*} \approx 0.5 \times 10^{12} \mathrm{~cm}^{-2}$ per surface (Fig. 1d), hence transport occurs through a landscape of electron and hole puddles with $\theta_{\mathrm{B}}$ spanning the entire range $0 \leq \theta_{\mathrm{B}} \leq \pi$. It appears that in this regime of highly inhomogeneous Berry phase that both WAL and WL (expected as $\theta_{\mathrm{B}} \rightarrow 0$ ) are suppressed.

\section{Discussion}

In contrast to semi-classical Boltzmann transport in $\mathrm{Bi}_{2} \mathrm{Se}_{3} \mathrm{TI}$ films, where the signature of finite-size effect appear only in the ultrathin limit ( $\leq 3 \mathrm{QL}$ ) (ref. 13), phase coherent transport offers an exquisitely sensitive probe of the hybridization of top and bottom transport channels, detecting a gap $\Delta$ as small as $0.3 \mathrm{meV}$ 
in the 12 QL TI film ${ }^{12}$, as well as destruction of topological protection in the few QL film. We note that similar suppression of WAL was recently observed in thin epitaxial $\mathrm{Bi}_{2} \mathrm{Se}_{3}$ of varying thickness (and $\Delta$ ) but fixed $E_{\mathrm{F}}$ (ref. 22), and a competition between WAL and WL was observed in gated 4 QL epitaxial $\left(\mathrm{Bi}_{0.57} \mathrm{Sb}_{0.43}\right)_{2} \mathrm{Te}_{3}$ (ref. 33). Our observation of the WAL by tuning both $E_{\mathrm{F}}$ and $\Delta$ (thickness) allows us to identify the ratio of $\tau_{\varphi} / \tau_{\mathrm{t}}$ as the driver for the crossover between coupled and decoupled surfaces, and the ratio of $\Delta$ to disorder strength as the driver for the crossover to the regime of suppressed WAL/WL. We expect that our results are important in identifying the regimes of carrier density and film thickness in which effects related to topological coherent transport can be observed in thin TI films.

\section{Methods}

$\mathrm{Bi}_{2} \mathrm{Se}_{3}$ field effect transistor fabrication. Low-doped (carrier density $\sim 10^{17} \mathrm{~cm}^{-3}$ ) bulk $\mathrm{Bi}_{2} \mathrm{Se}_{3}$ single crystals with bulk resistivity exceeding $2 \mathrm{~m} \Omega \mathrm{cm}^{-1}$ at $300 \mathrm{~K}$ were grown by melting high purity bismuth $(6 \mathrm{~N})$ and selenium $(5 \mathrm{~N})$ in sealed quartz ampoules ${ }^{23}$. Crystals were exfoliated with Scotch tape and deposited on doped $\mathrm{Si}$ covered with $300 \mathrm{~nm} \mathrm{SiO}_{2}$. Thin $\mathrm{Bi}_{2} \mathrm{Se}_{3}$ crystals with thickness ranging from 5 to $17 \mathrm{~nm}$ were identified by combined use of optical and atomic force microscopy. From the atomic force microscopy height profile of $\mathrm{Bi}_{2} \mathrm{Se}_{3}$ thin flakes, atomically flat surfaces with height variation $<1$ QL were chosen as channel area. Electron beam lithography, thermal evaporation and liftoff techniques were used to make electrical contact (Cr/Au: 5/70 nm). For accurate determination of the geometric factor, thin films were patterned into Hall bar geometry (see Fig. 1a) with typical aspect ratio $(L / W)$ of about 2 and shortest length exceeding $2 \mu \mathrm{m}$ using Ar plasma at a pressure of $\sim 6.7 \mathrm{~Pa}\left(5 \times 10^{-2} \mathrm{Torr}\right)$. Molecular charge transfer doping was done by thermal evaporation of $\sim 10 \mathrm{~nm}$ of 2,3,5,6-tetrafluoro-7,7,8,8-tetracyanoquinodimethane organic molecules (Aldrich) on top of the fabricated samples ${ }^{24}$.

Measurement. Transport measurements were performed using standard four-probe ac methods with low frequency $(<17 \mathrm{~Hz})$ excitation currents (r.m.s. amplitude $100 \mathrm{nA}$ ) using Stanford Research Systems SR830 Lock in amplifiers and a commercial cryostat equipped with $9 \mathrm{~T}$ superconducting magnet. For the $12 \mathrm{QL}$ device, five different WAL measurements as a function of gate voltage at $2 \mathrm{~K}$ were conducted, where the sample was warmed up to $300 \mathrm{~K}$ and exposed to air for a few hours between thermal runs.

\section{References}

1. Novoselov, K. S. et al. Two-dimensional gas of massless Dirac fermions in graphene. Nature 438, 197-200 (2005).

2. Qi, X. L. \& Zhang, S. -C. Topological insulators and superconductors. Rev Mod. Phys. 83, 1057-1110 (2011).

3. Hasan, M. Z. \& Kane, C. L. Colloquium: topological insulators. Rev. Mod. Phys. 82, 3045-3067 (2010)

4. Moore, J. Topological insulators: the next generation. Nat. Phys. 5, 378-380 (2009).

5. Zhang, H. J. et al. Topological insulators in $\mathrm{Bi}_{2} \mathrm{Se}_{3}, \mathrm{Bi}_{2} \mathrm{Te}_{3}$ and $\mathrm{Sb} 2 \mathrm{Te} 3$ with a single Dirac cone on the surface. Nat. Phys. 5, 438-442 (2009).

6. Hsieh, D. et al. A tunable topological insulator in the spin helical Dirac transport regime. Nature 460, 1101-1105 (2009).

7. Chen, Y. L. et al. Experimental realization of a three-dimensional topological insulator $\mathrm{Bi}_{2} \mathrm{Te}_{3}$. Science 325, 178-181 (2009).

8. Xia, Y. et al. Observation of a large-gap topological-insulator class with a single dirac cone on the surface. Nat. Phys. 5, 398-402 (2009).

9. Fu, L., Kane, C. L. \& Mele, E. J. Topological insulators in three dimensions. Phys. Rev. Lett. 98, 106803 (2007).

10. Hikami, S., Larkin, A. I. \& Nagaoka, Y. Spin-orbit interaction and magnetoresistance in the two dimensional random system. Prog. Theor. Phys. 63, 707-710 (1980).

11. Beenakker, C. W. J. \& Houten, H. V. Quantum transport in semiconductor nanostructures. Solid State Phys. 44, 1-228 (1991).

12. Zhang, Y. et al. Crossover of the three-dimensional topological insulator $\mathrm{Bi}_{2} \mathrm{Se}_{3}$ to the two-dimensional limit. Nat. Phys. 6, 584-588 (2010).

13. Cho, S., Butch, N. P., Paglione, J. \& Fuhrer, M. S. Insulating behavior in ultrathin bismuth selenide field effect transistors. Nano Lett. 11, 1925-1927 (2011).
14. Linder, J., Yokoyama, T. \& Sudbø, A. Anomalous finite size effects on surface states in the topological insulator $\mathrm{Bi}_{2} \mathrm{Se}_{3}$. Phys. Rev. B. 80, 205401 (2009).

15. Chen, J. et al. Gate-voltage control of chemical potential and weak antilocalization in $\mathrm{Bi}_{2} \mathrm{Se}_{3}$. Phys. Rev. Lett. 105, 176602 (2010).

16. Checkelsky, J. G., Hor, Y. S., Cava, R. J. \& Ong, N. P. Surface state conduction observed in voltage-tuned crystals of the topological insulator $\mathrm{Bi}_{2} \mathrm{Se}_{3}$. Phys. Rev. Lett. 106, 196801 (2010).

17. Kim, Y. S. et al. Thickness-dependent bulk properties and weak antilocalization effect in topological insulator $\mathrm{Bi}_{2} \mathrm{Se}_{3}$. Phys. Rev. B 84, 073109 (2011).

18. He, H.-T. et al. Impurity effect on weak antilocalization in the topological insulator Bi2Te3. Phys. Rev. Lett. 106, 166805 (2011).

19. Chen, J. et al. Tunable surface conductivity in $\mathrm{Bi}_{2} \mathrm{Se}_{3}$ revealed in diffusive electron transport. Phys. Rev. B 83, 241304 (2011).

20. Steinberg, H., Laloe, J. B., Fatemi, V., Moodera, J. S. \& Jarillo-Herrero, P. Electrically tunable surface-to-bulk coherent coupling in topological insulator thin films. Phys. Rev. B 84, 233101 (2011).

21. Matsuo, S. et al. Weak antilocalization and conductance fluctuation in a submicrometer-sized wire of epitaxial $\mathrm{Bi}_{2} \mathrm{Se}_{3}$. Phys. Rev. B 85, 075440 (2012).

22. Taskin, A. A., Sasaki, S., Segawa, K. \& Ando, Y. Manifestation of topological protection in transport properties of epitaxial $\mathrm{Bi}_{2} \mathrm{Se}_{3}$ thin films. Phys. Rev. Lett. 109, 066803 (2012)

23. Butch, N. P. et al. Strong surface scattering in ultrahigh-mobility $\mathrm{Bi}_{2} \mathrm{Se}_{3}$ topological insulator crystals. Phys. Rev. B 81, 241301 (2010).

24. Kim, D. et al. Surface conduction of topological Dirac electrons in bulk insulating $\mathrm{Bi}_{2} \mathrm{Se}_{3}$. Nat. Phys. 8, 460-464 (2012).

25. Beidenkopf, H. et al. Spatial fluctuations of helical Dirac fermions on the surface of topological insulators. Nat. Phys. 7, 939-943 (2011).

26. Adam, S., Hwang, E. H., Galitski, V. M. \& Das Sarma, S. A self-consistent theory for graphene transport. Proc. Natl Acad. Sci. USA 104, 18392-18397 (2007).

27. Adam, S., Hwang, E. H. \& Das Sarma, S. Two-dimensional transport and screening in topological insulator surface states. Phys. Rev. B 85, 235413 (2012).

28. Garate, I. \& Glazman, L. Weak localization and antilocalization in topological insulator thin films with coherent bulk-surface coupling. Phys. Rev. B 86, 035422 (2012)

29. Lu, H. Z., Shi, J. \& Shen, S. Q. Competition between weak localization and antilocalization in topological surface states. Phys. Rev. Lett. 107, 076801 (2011).

30. Cha, J. et al. Effects of magnetic doping on weak antilocalization in narrow $\mathrm{Bi}_{2} \mathrm{Se}_{3}$ nanoribbons. Nano Lett. 12, 4355-4359 (2012).

31. Tkachov, G. \& Hankiewicz, E. M. Weak antilocalization in HgTe quantum wells and topological surface states: Massive versus massless Dirac fermions. Phys. Rev. B 84, 035444 (2011).

32. Zhu, Z. H. et al. Rashba spin-splitting control at the surface of the topological insulator $\mathrm{Bi}_{2} \mathrm{Se}_{3}$. Phys. Rev. Lett. 107, 186405 (2011).

33. Lang, M. et al. Competing weak localization and weak antilocalization in ultrathin topological insulators. Nano Lett. 13, 48-53 (2013).

\section{Acknowledgements}

This work was supported by NSF grant number DMR-1105224. Preparation of $\mathrm{Bi}_{2} \mathrm{Se}_{3}$ was supported by NSF MRSEC (DMR-0520471) and DARPA-MTO award (N66001-09c-2067). N.P.B. was partially supported by the Centre for Nanophysics and Advanced Materials. M.S.F. acknowledges support from an ARC Laureate Fellowship. D.K. acknowledges useful conversations with Sergey S. Pershoguba.

\section{Author contributions}

D.K. fabricated devices, performed the electrical measurements and analysed the data with M.S.F. P.S., N.P.B. and J.P. prepared single crystal $\mathrm{Bi}_{2} \mathrm{Se}_{3}$ starting material. D.K. and M.S.F. wrote the manuscript with contributions from all authors.

\section{Additional information}

Supplementary Information accompanies this paper at http://www.nature.com/ naturecommunications

Competing financial interests: The authors declare no competing financial interests

Reprints and permission information is available online at http://npg.nature.com/ reprintsandpermissions/

How to cite this article: Kim, D. et al. Coherent topological transport on the surface of $\mathrm{Bi}_{2} \mathrm{Se}_{3}$. Nat. Commun. 4:2040 doi: 10.1038/ncomms3040 (2013). 